\title{
NLTE Line-Blanketed Model Stellar Atmospheres
}

\author{
Thierry Lanz ${ }^{1}$, Ivan Hubeny ${ }^{2}$ and Sara R. Heap \\ LASP, NASA Goddard Space Flight Center, Greenbelt, MD 20771
}

\begin{abstract}
.
We briefly review the assumptions and methods required to construct NLTE line-blanketed model atmospheres of hot stars. We describe our new grid of NLTE model atmospheres covering the parameter range of $\mathrm{O}$ stars at various metallicities. We have applied these new models to the analysis of HST/STIS and FUSE UV spectra of O stars in the SMC. This analysis leads us to revise down the effective temperature scale of O-type stars.
\end{abstract}

\section{Introduction}

The last decade saw dramatic improvements in our ability to model stellar atmospheres. At the same time, recent advances in observational detectors have resulted in unprecedented quality (and quantity) of stellar spectra leading to a renewed need and interest for better model atmospheres. Stellar atmosphere theory and modeling are thus enjoying a period of revival. On the modeling side, the central role in the development of efficient numerical techniques has been played by Accelerated Lambda Iteration (ALI) methods. We discuss ALI methods in Sect. 3 only briefly, since they are reviewed by Werner in this volume. Another review was recently written by Hubeny (2002), and the specific application of ALI to modeling stellar atmospheres is discussed by Hubeny \& Lanz (2002).

We will concentrate here on the topic of NLTE line blanketing in 1-D, plane-parallel, hydrostatic atmospheres, i.e. stellar photospheres. In particular, we will discuss our implementation of NLTE line-blanketing within our model atmosphere code, TLUSTY, and its applications to hot stars. Spherical expanding atmospheres are discussed in this volume by Baron et al. and by Bennett in the context of cool stars. This was also extensively covered during the recent Tuebingen workshop on stellar atmospheres (Hubeny, Mihalas, \& Werner 2002) by several contributors (Hamann; Koesterke; Hillier; Höflich; Hauschildt).

\section{Model assumptions}

Model assumptions that are made to construct model stellar atmospheres may be ranged into five broad categories:

\footnotetext{
${ }^{1}$ Department of Astronomy, University of Maryland, College Park, MD 20742

${ }^{2}$ National Optical Astronomy Observatories, Tucson, AZ 85726
} 
- Geometry: The problem is greatly simplified by adopting a 1-D geometry (plane-parallel or spherical geometry). An exact solution of radiative transfer in a more complex geometry requires many additional spatial points, but also many more rays to solve accurately for scattering. The problem quickly becomes excessively costly. Multi-dimensional radiative transfer is solved today for optically-thin and optically-thick plasmas, but is still in its infancy as far as stellar atmospheres (and accretion disks) are concerned. For some recent progress in multi-D radiative transfer in stellar (solar) atmospheres, see e.g., Carlsson (this volume), Asplund et al. (2000) and van Noort et al. (2002).

- Dynamics: Extensive grids of model stellar atmospheres have been computed assuming static atmospheres (Kurucz 1993, Gustafsson et al. in this volume, and this paper). In modeling dynamical situations, a full range of assumptions is possible, from prescribed velocity laws, that are used in most analyses of stellar winds, to solving the full hydrodynamical problem. Examples of the latter include the case of line formation in a convective atmosphere (Asplund et al. 2000), winds of massive stars (Pauldrach et al. 2001), instabilities in radiatively-driven winds (Runacres \& Owocki 2002), or spectral evolution of supernovae (Höflich et al. 2002).

- Thermodynamics: Assuming LTE greatly reduces the cost of calculating model stellar atmospheres, because the source function and the opacities depend on the local temperature and density only. If this assumption is invalid however, one needs to solve the statistical equilibrium equations for all species and therefore compute all necessary radiative rates. Additionally, an energy conservation equation must be solved. Most often, radiative equilibrium is assumed, but a more general energy equation should be considered when modeling the chromosphere and corona of cool stars or stellar winds.

- Composition: The composition of the atmospheric plasma will determine the plasma opacity, and thus the transport of radiative energy in the atmosphere and the emergent spectrum. The problem may be simplified by assuming a very simple composition (e.g., an hydrogen-helium atmosphere), resulting in easily-describable opacities and a limited system of statistical equilibrium equations when LTE is not assumed. Statistical approaches, like Opacity Distribution Functions and Opacity Sampling, have been devised to incorporate complicated opacities from realistic plasma compositions. For some applications, see among others, Kurucz (1979), Hauschildt et al. (1997), Hillier \& Miller (1998), or this paper.

- Electromagnetic Fields: Most studies assume a global electric neutrality of the medium and neglect magnetic fields.

Although progress in the field of stellar atmosphere modeling has been quite rapid during the last decade thanks to new numerical methods and faster computers, we are still not reaching the point of having the necessary computing resources to solve the complete problem, namely the full 3-D radiation magnetohydrodynamics with detailed NLTE opacities. We must therefore adopt some 
of the restrictive assumptions listed above while aiming still for realistic model atmospheres that best match high-quality spectroscopic data. Multi-D geometries represent the biggest modeling challenge for the coming years but, at the present time, we must accept a restriction to 1-D models because of the very coarse frequency sampling achievable in multi-D models. We argue now that a most important point in stellar atmosphere modeling is to strive for the best description of the interaction between matter and radiation, viz. NLTE lineblanketing. We discuss this topic in the next section, before addressing the question of the relevance of static photosphere models of hot stars.

\subsection{NLTE line blanketing}

Local Thermodynamic Equilibrium breaks down at some point in every stellar atmosphere. On one hand, collisions between particles favor LTE; on the other hand, the non-local radiation field and the photon losses to the interstellar medium are the reasons while LTE cannot hold. Therefore, LTE will be a valid assumption only when collisional processes dominate the radiative processes. The net collisional rate in every atomic transition must be much larger than the net radiative rate to achieve LTE. The largest departures from LTE are expected in hot and luminous stars, but departures from LTE also occur in main-sequence stars, in solar-type stars (e.g. in their chromosphere), even in planetary atmospheres.

The most important NLTE effect in stellar atmospheres is overionization relative to LTE ionization. The ionization structure depends on the radiative and collisional ionization rates. While the latter is function of the local temperature, the former depends on the radiation field which is emitted in deeper, hotter layers. This results in ionization rates that are larger than their LTE counterparts. To estimate correctly the NLTE ionization structure, one requires accurate mean intensities which in turn require an accounting of all sources of opacity. When neglecting in particular the opacity of Fe lines, NLTE models predict too strong EUV/UV radiation fields, and therefore tend to overestimate the ionization and the importance of departures from LTE. Results from NLTE calculations neglecting background opacities must thus be viewed with caution.

Calculating reliable and realistic NLTE models is quite costly, and typically requires $10^{4}$ times more CPU cycles than line-blanketed LTE models do. It is therefore essential to assess when NLTE models are necessary by determining the extent of departures from LTE for different stellar spectral types and for different wavelength regions or spectral features. High-quality spectroscopic data are now being obtained routinely, and their interpretation requires models of similar quality or, at the very least, we must show that LTE models are accurate enough to represent adequately these observations.

\subsection{Photospheric vs. Wind models}

It is well-known that $\mathrm{O}$ star spectra are characterized by the signature of strong, fast winds. Therefore, could photospheric models assuming a plane-parallel geometry in hydrostatic equilibrium still be useful? The answer is positive for two major reasons:

1. Most spectral lines (excluding strong resonance lines) are formed in the photosphere where velocities are small and geometrical extension is negli- 

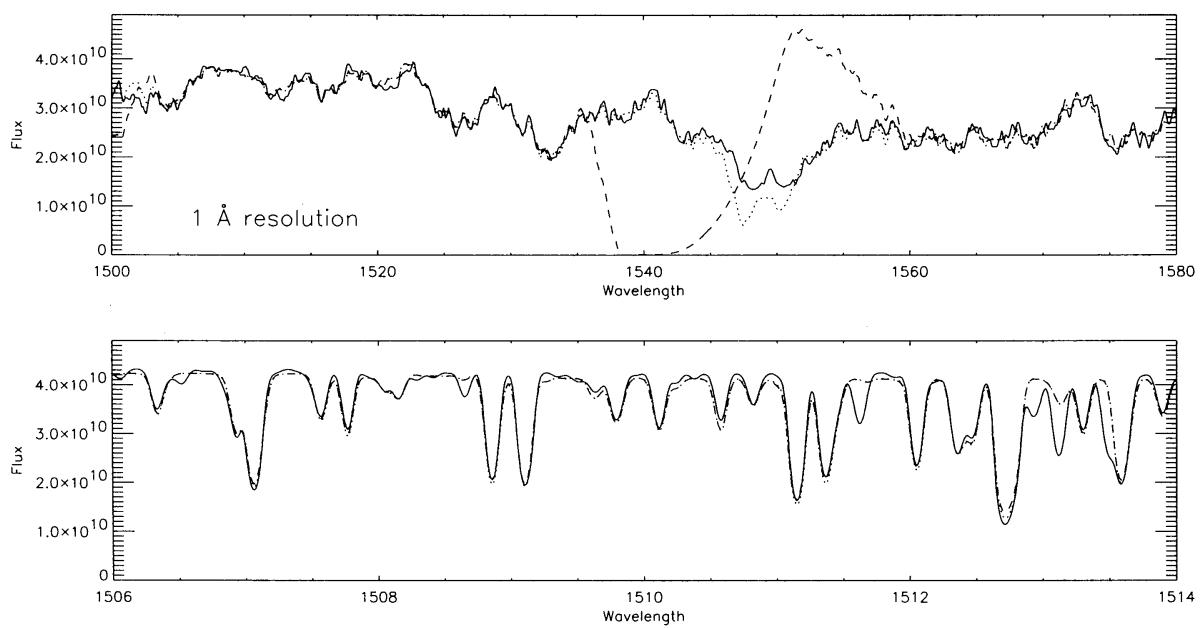

Figure 1. A comparison of three theoretical spectra calculated with TLuSTY (full line) and CMFGEN (dashed: $\dot{M}=310^{-7} M_{\odot} /$ yr; dotted: $\dot{M}=10^{-10} M_{\odot} / \mathrm{yr}$ ) for a $T_{\text {eff }}=35000 \mathrm{~K}, \log g=4.0$, solar composition model. The hydrostatic density structure calculated by TLUSTY was adopted in the deep layers of the CMFGEN models. The spectra are very similar, except the $\mathrm{CIV}$ resonance lines which are formed in the stellar wind. A $1 \AA$ resolution smoothing has been applied to the top panel's spectra. Some minor differences can be attributed to differences in atomic data (e.g. $\lambda 1513)$.

gible. Indeed, the spectrum of slowly-rotating $\mathrm{O}$ stars show mostly narrow and symmetric lines. This statement is valid in all but the most extreme cases of stellar winds, like those observed in Wolf-Rayet stars and Of supergiants. Moreover, winds are weaker in low-metallicity environments making photospheric models even more relevant in this case. We compare in Fig. 1 spectra calculated with two NLTE model atmosphere codes: the wind code CMFGEN (Hillier \& Miller 1998) and the photospheric code TLUSTY (Hubeny \& Lanz 1995). Both codes incorporate detailed NLTE line opacities. We have chosen a test case, $T_{\text {eff }}=35000 \mathrm{~K}, \log g=4.0$, $R=8.3 R_{\odot}, M=25 M_{\odot}, L=93000 L_{\odot}, \xi_{\mathrm{t}}=10 \mathrm{~km} / \mathrm{s}$, and a solar composition. Two CMFGEN models have been calculated, assuming a mass loss rate typical of these stellar parameters $\left(\dot{M}=310^{-7} M_{\odot} /\right.$ yr $)$ and low mass loss rate $\left(\dot{M}=10^{-10} M_{\odot} /\right.$ yr $)$. Fig. 1 shows that the predicted spectra are almost identical, except for the C IV doublet which is formed in the wind. All the other lines are formed in the quasi-static photosphere. See Hillier \& Lanz (2001) for more details about this comparison.

2. While the paradigm of radiatively-driven winds is well established, many uncertainties remain on the exact properties of stellar winds. First, we do not have in many cases a consistent solution: the calculated line force is often too small to drive a wind (though some progress is being made), so a $\beta$ - 
type velocity law must be adopted to describe empirically the velocity and density structure of the wind. Second, radiative equilibrium is not really satisfied in these winds, where shocks dump mechanical energy and heat the wind, thus changing its ionization structure (e.g., super-ionization). Finally, the assumption of one-dimensional geometry is challenged by the likely presence of dense clumps in the wind; the role of magnetic fields is not yet understood, and their importance in structuring the wind will very likely be more important than in the photosphere.

The determination of the fundamental parameters of massive stars will be therefore on much safer grounds if we could use selected photospheric lines and thus avoid all uncertainties remaining with wind models. However, we have to show that the selected lines are not affected by the wind and we must understand the limits of validity of this approach.

\section{Methods}

\subsection{Complete Linearization}

The basic structural equations are discretized in frequency and depth, which yield a set of highly-coupled, non-linear algebraic equations. The fundamental problem of stellar atmosphere modeling is to find a robust and efficient method for solving these equations. The decisive breakthrough, and in fact the beginning of the modern era of stellar atmosphere models, was the development of the complete linearization (CL) method by Auer \& Mihalas (1969). This was the first scheme to treat all equations on the same footing, thus solving all structural equations simultaneously. Before that, the equations were typically solved one at a time, iterating among them. In many cases, iterations were slow, or a scheme failed to converge at all.

The original complete linearization is nothing other than the NewtonRaphson method. This method requires to invert the Jacobi matrix (Jacobian) of the system of equations, where the $i j$-element of the Jacobian is the derivative of the $i$-th equation with respect to the $j$-th unknown. It is immediately clear that the original complete linearization, despite its inherent power and robustness, cannot be used as a general numerical scheme because in realistic calculations one must use a very large number of frequency points to describe the radiation field sufficiently accurately - of the order of $10^{5}$ to $10^{6}$ points. Inverting matrices of this dimension is completely out of question. One obviously must seek less global, but much faster schemes.

\subsection{Accelerated Lambda Iteration}

The Accelerated Lambda Iteration (ALI) method (see, e.g., Werner in this volume, Hubeny 2002, and references therein) is a simple yet very powerful numerical scheme that has revolutionized the field of stellar atmospheres and radiative transfer. ALI drastically reduces the number of unknowns by eliminating the mean intensity of radiation at all frequencies from the set of unknowns. This is achieved by expressing the mean intensity of radiation as

$$
J_{\nu}^{(n)}=\Lambda_{\nu}^{*} S_{\nu}^{(n)}+\left(\Lambda_{\nu}-\Lambda_{\nu}^{*}\right) S_{\nu}^{(n-1)},
$$


where $\Lambda_{\nu}$ and $\Lambda_{\nu}^{*}$ are the exact and the approximate Lambda operator, and $S_{\nu}$ the source function, all at frequency $\nu$. The superscript $n$ indicates the iteration number. The mean intensity of radiation is thus represented by two terms. The second one - the "correction" term - is known from the previous iteration and is thus not linearized, while the first one represents an action of an approximate (and, therefore, simple) operator, $\Lambda^{*}$, on the source function, which is expressed as a function of temperature, density, and atomic level populations. The radiative transfer equations are thus eliminated from the coupled system of structural equations. The method was first applied in the context of stellar atmosphere models by Werner (1986); a different variant, called "hydrid Complete Linearization/Accelerated Lambda Iteration" method, was developed by Hubeny \& Lanz (1995).

\subsection{Programs TLUSTY and SYNSPEC}

TLuSTY and SynsPEC form an user-oriented package for modeling stellar atmospheres and for stellar spectroscopic diagnostics. TLUSTY is a NLTE model atmosphere code, assuming radiative equilibrium, hydrostatic equilibrium, and plane-parallel geometry. TLUSTY uses an hybrid Complete Linearization / Accelerated Lambda Iteration Method (CL/ALI). This method builds on the advantages of ALI and Complete Linearization. Through ALI, the mean intensities of the radiation field are eliminated from the set of linearized structural equations. However, the hybrid CL/ALI method still allows us to linearize the radiation intensity at a few selected key frequencies, improving notably the convergence rate compared to a "pure" ALI approach. By reducing drastically the number of equations in the linearized set, we can incorporate consistently thousands of NLTE levels and the effect of millions of individual lines in the model atmospheres (Hubeny \& Lanz 1995). SYNSPEC computes the detailed emergent spectrum using a model atmosphere and the NLTE populations calculated with TLusty, and extensive linelists. These codes are user-oriented in the sense that all opacities included in the models are specified by the users, making thus TLUSTY and SYNSPEC very flexible tools for analyzing spectra of widely different objects from the X-ray domain to the infrared.

\subsection{Handling of atomic data}

Calculating realistic NLTE model stellar atmospheres requires data for radiative and collisional bound-bound, bound-free, and free-free transitions, for all species that are significant opacity sources. This may also include autoionization processes, di-electronic recombination, and Auger ionization. Generally, a high accuracy (better than 20\%) is desirable for radiative data because the reliability of the predicted emergent spectra and of the derived spectral diagnoses are directly influenced by the quality of the radiative data. On the other hand, moderate accuracy (let say, a factor 2 or better) is sufficient for collisional data which will determine by how much the level populations depart from their LTE values. Extensive atomic datasets are now available for radiative data, while the set of collisional data remains more limited requiring the use of generic formulae.

The bulk of the radiative data used in TLUSTY are extracted from Topbase, the database of the Opacity Project (OP 1995; Cunto et al. 1993). OP provides very extensive datasets of levels energies, $g f$-values, and photoionization cross- 
sections, that have been obtained from ab-initio calculations for all ions of the most abundant light species $(Z \leq 14, \mathrm{~S}, \mathrm{Ca}$, and $\mathrm{Fe}$ ); see Nahar (2002) for a description of the current status of this project and its continuation as the Iron Project. OP claims that these data are accurate at the $10 \%$ level or better. Level energies measured in laboratory experiments are extracted from the NIST database to update the theoretical energies. The second extensive dataset is extracted from Kurucz' web site and CD-ROMs (Kurucz 1994). His large semiempirical calculations for iron-peak elements provide the level energies and line data for our model atoms of $\mathrm{Fe}$ and $\mathrm{Ni}$ in Tlusty, while we use an updated version of his line list with SYNSPEC.

The NIST and the OP databases typically list tens to a few hundred individual energy levels for each ion of light elements $(Z<20)$, while the Kurucz data may contain over 10000 levels for some individual ions of iron-peak elements. Therefore, a different approach must be adopted for these two cases and a statistical approach is required in case of very large number of levels. For most ions of light elements, all levels below the ionization limit can generally be incorporated in the model atoms, while levels above the limit are assumed to be in LTE with respect to the ground state of the next ion. The populations of the latter levels are accounted for by means of the LTE partition function. We have applied the concept of superlevels to $\mathrm{Fe}$ and $\mathrm{Ni}$ ions. The underlying assumption is that the populations of individual levels inside a given superlevel follow the Boltzmann distribution. While the populations of superlevels could depart from their LTE values, all sublevels in a given superlevel share the same $b$-factor. To make sure that this is a reasonable approximation, one should group levels with very close excitation energies (thus having large collisional rates between levels) in the superlevel. This applies therefore quite well also to grouping high-excitation levels of light elements. This concept was first introduced by Anderson (1989), and developed further by Dreizler \& Werner (1993) and Hubeny \& Lanz (1993). Detailed expressions for the mean energies of superlevels, statistical weights, and transition cross-sections between superlevels, may be found in Hubeny \& Lanz (1995). We have adopted a second criterion besides energy when grouping levels: we demand that all levels in a given superlevel share the same parity. This avoids radiative transitions within a superlevel; and, more importantly, this enhances the validity of the assumption of equal $b$-factors because parity will be accounted correctly for radiative transitions between superlevels and the ground state of the ion (i.e., metastable states and transitions out and into them are treated properly). We have built model atoms in which all level data calculated by Kurucz (typically, a few thousand and up to 13000 levels per ion) are grouped into about 40-60 superlevels per ion. Grotrian diagrams of Fe IV and Fe V are displayed in Fig. 2.

The absorption cross-section of a superline between two superlevels can be quite complex, involving hundreds or thousands of individual lines. A detailed representation may require a very large number of frequency points. Two approaches have been implemented to limit the number of frequencies. First, we used Opacity Distribution Functions (ODFs): the total opacity from all lines in a given transition (computed using Voigt profiles for each line) is sorted; the sorted opacities could be represented with a limited number of frequencies, typically 15 to 30 per transition, or 30000 to 50000 frequencies for the whole spectrum. Exact details of line blends are lost; some care should thus be exercised in checking 

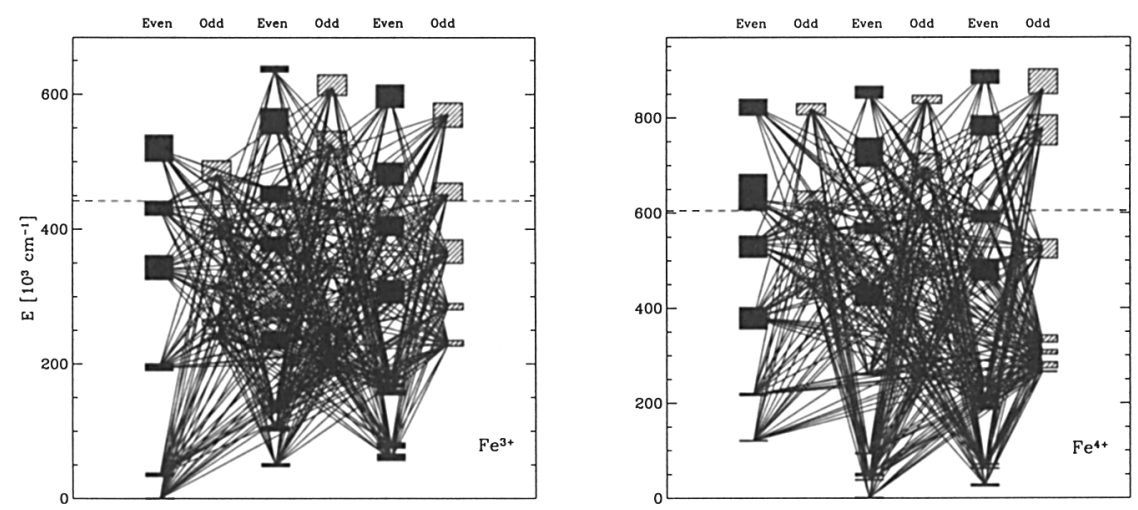

Figure 2. Fe IV and Fe V model atoms: Superlevels are represented by boxes (full: even parity; shaded: odd parity) with a vertical extension indicating the range of level energies. The different columns have no special meaning but have been set for clarity; the horizontal dashed lines show the ionization energy.

that unintentional blends between strong lines of light elements and Fe ODFs are not affecting the results. The second method is Opacity Sampling (OS) which is a simple Monte Carlo-like sampling of the superline cross-sections. It has the advantage of treating blends exactly. However, the cores of important strong lines might be missed if too few frequency points are used. We use a variant of OS, sampling the whole spectrum at prescribed intervals in frequency. Our model atmospheres are sampled with a typical step of 0.75 Doppler widths, using thus close to 200000 frequency points for an $\mathrm{O}$ star model. While costly in terms of computing requirements, we can thus sample the spectrum with a sufficient resolution to ensure that every line is accounted for. This is the current standard mode in TLusTY. We can adopt a larger step (30 Doppler widths) to represent the opacity of iron lines and thus decrease the number of frequency points to about 60000 ; in this case, however, we keep a small frequency step to sample accurately the lines of light elements (e.g. the Si IV lines).

Fig. 3 illustrates how the radiation field is represented by the two approaches. The differences between ODF and OS models regarding blends between the $\mathrm{Fe}$ and the Si IV lines is quite apparent. The different approach (ODF vs. OS) results in a larger change in the temperature stratification compared to the adoption of a larger sampling step. We interpret this as reflecting incorrect blends between ODFs and other lines.

Detailed photoionization cross-sections are extracted from the OP database. Most cross-sections have a complicated structure with many autoionization resonances. An examination of the cross-sections reveals however that the sharp resonances are not fully resolved by the OP calculations. Moreover, theoretical energies have an accuracy of $2-3 \%$, and the resonances are therefore shifted off their exact wavelengths. Following Bautista et al. (1998), we have therefore adopted the concept of resonance-averaged cross-sections. The cross-sections are 

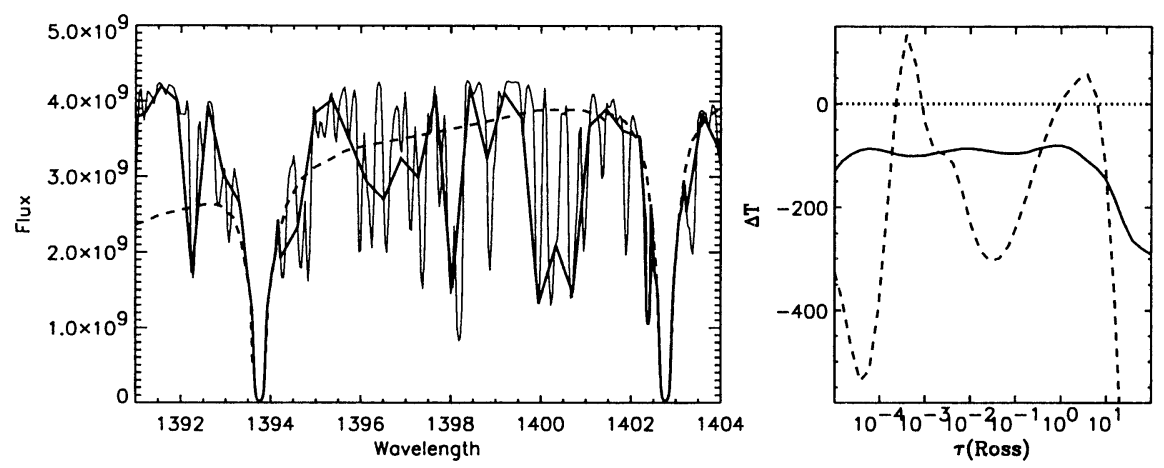

Figure 3. ODF and OS in TLuSTY: UV spectrum of a 35000/4.0 model atmosphere with a zoom on the region of the Si IV resonance lines; two sampling steps are shown: 0.75 (thin) and 30 (thick) Doppler widths, as well as the ODF model (dashed). The resulting effect on the temperature structure is displayed in the right panel, relative to the OS/0.75 model: OS/30 model (full line), and ODF model (dashed line).

convolved with a Gaussian that smoothes the sharp resonances, with a width, $\delta E / E=0.03$, corresponding to the typical uncertainty of the resonance positions. This approach thus addresses the two problems of the original OP cross-sections. See Lanz \& Hubeny (2002a) for additional details on atomic data handling in TLUSTY.

\section{A grid of NLTE model atmospheres of $O$ stars}

With our NLTE model atmosphere code, TLUSTY, we have built a grid of over 450 NLTE line-blanketed model atmospheres covering the stellar parameter range of O-type stars at 7 different chemical compositions. The model atmospheres are calculated assuming a plane-parallel geometry, radiative and hydrostatic equilibria, and incorporate about 100,000 NLTE atomic levels of over 40 ions of $\mathrm{H}, \mathrm{He}, \mathrm{C}, \mathrm{N}, \mathrm{O}, \mathrm{Ne}, \mathrm{Si}, \mathrm{P}, \mathrm{S}, \mathrm{Fe}$, and $\mathrm{Ni}$. The levels are grouped into about 900 superlevels, with individual energy levels following Boltzmann statistics inside a superlevel. A total of 8000 lines of the light elements and about 2 millions lines of Fe III-VI and Ni III-VI are accounted for in the calculations. Based on a line strength criterion, $\mathrm{Fe}$ and $\mathrm{Ni}$ lines are dynamically selected from a total of 12 million lines listed by Kurucz (1994). The line opacity is represented by a variant of Opacity Sampling, adopting a small sampling step (0.75 Doppler widths), using close to 200000 frequency points over the whole spectrum.

We have covered the parameter space of O-type stars, with effective temperature ranging from $27,500 \mathrm{~K}$ to $55,000 \mathrm{~K}$ with a $2,500 \mathrm{~K}$ step, and the surface gravity in the range $3.0 \leq \log g \leq 4.75$ with a step of 0.25 dex (see Table 1). Models at high temperatures and low surface gravities are becoming physically 
Table 1. Range of stellar parameters $\left(T_{\text {eff }} \times 10^{-3} \mathrm{~K}, \log g\right)$ in the model grid.

\begin{tabular}{l|cccccccccccc}
\hline & 27.5 & 30 & 32.5 & 35 & 37.5 & 40 & 42.5 & 45 & 47.5 & 50 & 52.5 & 55 \\
\hline 3.00 & $\mathbf{X}$ & $\mathbf{X}$ & & & & & & & & & & \\
3.25 & $\mathbf{X}$ & $\mathbf{X}$ & $\mathbf{X}$ & $\mathbf{X}$ & & & & & & & & \\
3.50 & $\mathbf{X}$ & $\mathbf{X}$ & $\mathbf{X}$ & $\mathbf{X}$ & $\mathbf{X}$ & $\mathbf{X}$ & & & & & & \\
3.75 & $\mathbf{X}$ & $\mathbf{X}$ & $\mathbf{X}$ & $\mathbf{X}$ & $\mathbf{X}$ & $\mathbf{X}$ & $\mathbf{X}$ & $\mathbf{X}$ & & & & \\
4.00 & $\mathbf{X}$ & $\mathbf{X}$ & $\mathbf{X}$ & $\mathbf{X}$ & $\mathbf{X}$ & $\mathbf{X}$ & $\mathbf{X}$ & $\mathbf{X}$ & $\mathbf{X}$ & $\mathbf{X}$ & $\mathbf{X}$ & $\mathbf{X}$ \\
4.25 & $\mathbf{X}$ & $\mathbf{X}$ & $\mathbf{X}$ & $\mathbf{X}$ & $\mathbf{X}$ & $\mathbf{X}$ & $\mathbf{X}$ & $\mathbf{X}$ & $\mathbf{X}$ & $\mathbf{X}$ & $\mathbf{X}$ & $\mathbf{X}$ \\
4.50 & $\mathbf{X}$ & $\mathbf{X}$ & $\mathbf{X}$ & $\mathbf{X}$ & $\mathbf{X}$ & $\mathbf{X}$ & $\mathbf{X}$ & $\mathbf{X}$ & $\mathbf{X}$ & $\mathbf{X}$ & $\mathbf{X}$ & $\mathbf{X}$ \\
4.75 & $\mathbf{X}$ & $\mathbf{X}$ & $\mathbf{X}$ & $\mathbf{X}$ & $\mathbf{X}$ & $\mathbf{X}$ & $\mathbf{X}$ & $\mathbf{X}$ & $\mathbf{X}$ & $\mathbf{X}$ & $\mathbf{X}$ & $\mathbf{X}$ \\
\hline \hline
\end{tabular}

unstable close to the Eddington limit and have therefore not been calculated. A total of 68 models are available for each chemical composition. Our grid covers compositions from solar down to $1 / 100$ times solar in 7 steps: solar, $1 / 2$, $1 / 5,1 / 10,1 / 30,1 / 50$, and $1 / 100$ times solar. Abundances are scaled to the solar composition. In all cases, we have however assumed a solar helium abundance, $\mathrm{He} / \mathrm{H}=0.1$ by number. TLUSTY can readily accommodate non solarscaled abundances resulting, e.g., from mixing of CNO-cycle processed material; however, this would increase very quickly the total number of models to compute in the grid by increasing the dimensionality of the parameter space. Thus, we view this grid as a starting point from which detailed abundance analyses can be performed. Finally, we have always assumed a microturbulent velocity, $\xi_{\mathrm{t}}=10 \mathrm{~km} / \mathrm{s}$, which is the typical value found in spectral analyses of $\mathrm{O}$ stars. The resulting line desaturation mimics the effect of a small velocity gradient found at the base of a stellar wind.

The models (vertical structure, spectrum), as well as the current version of the computer code and the atomic data, are available from our Web site (http://tlusty.gsfc.nasa.gov). A detailed description of the models and their properties will be published elsewhere (Lanz \& Hubeny 2002b).

\section{Effective temperature scale of O-type stars}

High-quality, high-resolution spectra of 17 O-type stars in the Small Magellanic Cloud have been secured in the far-UV with HST/STIS and FUSE (for a subset only) and in the optical at ESO and AAT, in order to characterize the properties of massive stars in a low-metallicity environment. Walborn et al. (2000) discussed the objectives and first results of this program. We have applied our new grid of NLTE model atmospheres to the analysis of this sample to derive the basic stellar parameters. Here, we report on our preliminary results on the effective temperature scale. The HeI/He II ionization balance is the usual spectroscopic $T_{\text {eff }}$ estimator in $\mathrm{O}$ stars. However, in many cases, the optical He I lines are masked by nebular emission. Ionization balance of metal lines in the UV (C III 1176/ C IV 1169; $\mathrm{N}$ III 980/N IV 1718; O IV 1338-43/O V 1371; S IV 1073/S v 1502; Fe IV/Fe V/Fe VI) provide then a valuable alternative. Fig. 4 illustrates the case of carbon ionization balance. Although both C III and C IV lines grow stronger with higher surface gravity, abundance, or microturbulent 

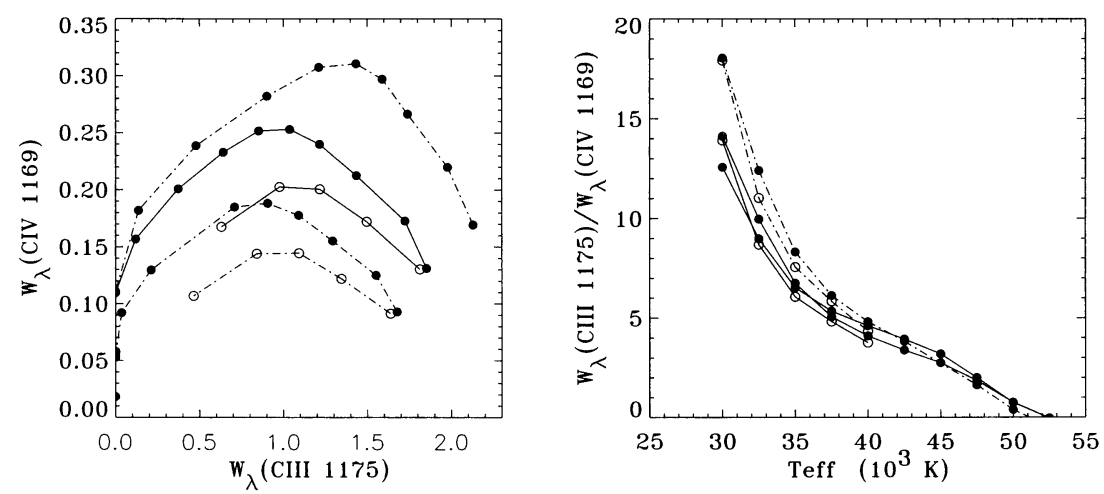

Figure 4. Effective temperatures of $\mathrm{O}$ stars from the carbon ionization ratio. The left panel illustrates the sensitivity of the line strengths with gravity (empty: $\log g=3.5$; filled: $\log g=4.0$ ), carbon abundance and microturbulent velocity (all curves with $10 \mathrm{~km} / \mathrm{s}$, but the top one with $20 \mathrm{~km} / \mathrm{s}$ ). The right panel shows however that the line strength ratio is mostly sensitive to $T_{\text {eff }}$.

velocity, the ratio of their line strengths is most sensitive to temperature (right panel) and provides a good $T_{\text {eff }}$ estimator.

We compare in Fig. 5 our temperatures to Vacca et al.'s (1996) calibration for $\mathrm{O} 4$ to $\mathrm{O} 9.7$ stars. Vacca et al.'s calibration is mostly based on analyses of the helium optical lines made in the 1980's and early 1990's with NLTE model atmospheres which incorporated only hydrogen and helium. We found that our new temperatures are systematically lower by about $10 \%$. We attribute this systematic difference to two compounding factors. First, fully line-blanketed model atmospheres result in deriving lower $T_{\text {eff }}$ due to the backwarming effect. However, we performed some tests and found that this would explain only half the difference even in galactic O stars. Second, the optical He I lines may be filled by wind emission (Sellmaier et al. 1993) or masked by nebular emission; however, the selected UV lines are less or not affected by these two effects. Using only the optical He I lines may thus lead to an overestimate of $T_{\text {eff }}$.

\section{Conclusions}

Extensive sets of atomic data can now be routinely incorporated in NLTE model stellar atmospheres. We are thus able to take advantage of the latest sophisticated atomic calculations to improve our description of the total opacities as well as of radiative rates. Generally, these models reproduce well most details of high-quality UV and optical spectra of hot stars. In particular, we point out that consistent fits of lines of several ions of different species can be achieved for main-sequence $\mathrm{O}$ stars, hot subdwarfs, and hot metal-rich white dwarfs. This indicates that the ionization structure of the model atmospheres is basically correct, showing thus that the claim of high accuracy of OP data (seen as a whole set) is well justified and that our handling of these data (Opacity Sampling, Resonance-Averaged Photoionization cross-sections) is also adequate. 

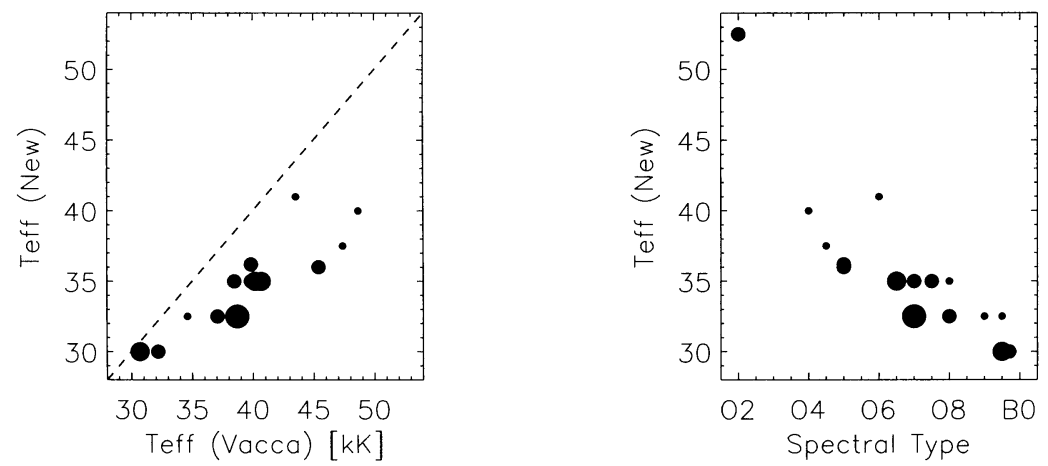

Figure 5. Our new effective temperatures compared to values derived from Vacca et al.'s (1996) calibration (left) and our new $T_{\text {eff }}$-spectral type relation (right). The point sizes are indicative of the luminosity class.

We have built an extensive grid of NLTE model atmospheres covering the parameter range of $\mathrm{O}$ stars. We have used these models to analyze high-quality STIS and FUSE UV spectra of SMC O stars. We found that the effective temperature scale of $\mathrm{O}$ stars needs to be revised downwards.

Acknowledgments. As a community, we are greatly indebted to the physicists who provided, and are still providing, all the necessary atomic data, in particular the OP team, Bob Kurucz, and the NIST team. The present sophistication of current model atmospheres would simply not been achievable without all their work during many years. This work was supported by NASA ADP, HST GO 7437, HST AR 7985, and FUSE B134 grants.

\section{Discussion}

COHEN: As $T_{\text {eff }}$ decreases, the radiative rates decrease and presumably the importance of non-LTE models decreases, there are significant uncertainties in non-LTE models due to uncertainties in atomic physics parameters. At what point does the second effect become comparable to the difference between LTE and non-LTE models.

LANZ: Indeed; at lower $T_{\text {eff }}$, the radiative rates decreases and we approach LTE. Then, the total rates are dominated by collisional rates. Errors in the radiative rates become thus less and less important. We always check that we do recover LTE in situations where LTE should prevail.

\section{References}

Anderson, L. S. 1989, ApJ, 339, 558

Auer, L. H., \& Mihalas, D. 1969, ApJ, 158, 641 
Asplund, M., Nordlund, A., Trampedach, R., Allende Prieto, C., \& Stein, R. F., 2000, A\&A, 359, 729

Bautista, M.A., Romano, P., \& Pradhan, A. K. 1998, ApJS, 118, 259

Cunto, W., Mendoza, C., Ochsenbein, F., \& Zeippen, C. J. 1993, A\&A, 275, L5

Dreizler, S., \& Werner, K. 1993, A\&A, 278, 199

Hauschildt, P. H., Baron, E., \& Allard, F. 1997, ApJ, 483, 390

Hillier, D. J., \& Lanz, T. 2001, in Spectroscopic Challenges of Photoionized Plasmas, Eds. G. Ferland \& D. W. Savin, ASP Conf. Ser., 247, 343

Hillier, D. J., \& Miller, D. L. 1998, ApJ, 496, 407

Höflich, P., Gerardy, C. L., Fesen, R. A., \& Sakai, S. 2002, ApJ, 568, 791

Hubeny, I. 2002, to appear in Stellar Atmospheric Modeling, Eds. I. Hubeny et al., ASP Conf. Ser.

Hubeny, I., \& Lanz, T. 1993, in Peculiar versus Normal Phenomena in A-type and Related Stars, Proc. IAU Coll. 138, Eds. M. M. Dworetsky et al., ASP Conf. Ser., 44, 98

Hubeny, I., \& Lanz, T. 1995, ApJ, 439, 875

Hubeny, I., \& Lanz, T. 2002, to appear in Stellar Atmospheric Modeling, Eds.

I. Hubeny et al., ASP Conf. Ser.

Hubeny, I., Mihalas, D., \& Werner, K. 2002, Stellar Atmospheric Modeling, ASP Conf. Ser. (in press)

Kurucz, R. L. 1979, ApJS, 40, 1

Kurucz, R. L. 1993, ATLAS9 Stellar Atmosphere Programs and $2 \mathrm{~km} / \mathrm{s}$ Grid, CD-ROM 13 (Cambridge, Mass: SAO)

Kurucz, R. L. 1994, Atomic Data for Fe and Ni, CD-ROM 22; Atomic Line Data, CD-ROM 23 (Cambridge, Mass: SAO)

Lanz, T., \& Hubeny, I. 2002a, to appear in Stellar Atmospheric Modeling, Eds. I. Hubeny et al., ASP Conf. Ser.

Lanz, T., \& Hubeny, I. 2002b, to be submitted to ApJ

Nahar, S. 2002, to appear in Stellar Atmospheric Modeling, Eds. I. Hubeny et al., ASP Conf. Ser.

The Opacity Project Team, 1995, The Opacity Project, Vol. 1 (Bristol, UK: Inst. of Physics Publications)

Pauldrach, A. W. A., Hoffmann, T. L., \& Lennon, M. 2001, A\&A, 375, 161

Runacres, M. C., \& Owocki, S. P. 2002, A\&A, 381, 1015

Sellmaier, F., Puls, J., Kudritzki, R. P., Gabler, A., Gabler, R., \& Voels, S. A. 1993, A\&A, 273, 533

Vacca, W. D., Garmany, C. D., \& Shull, J. M. 1996, ApJ, 460, 914

van Noort, M., Hubeny, I., \& Lanz, T. 2002, ApJ, 568, 1066

Walborn, N. R., Lennon, D. J., Heap, S. R., Lindler, D. J., Smith, L. J., Evans, C. J., \& Parker, J. W. 2000, PASP, 112, 1243

Werner, K. 1986, A\&A, 161, 177 